

А. В. Арутюнов, Накрывание нелинейных отображений на конусе в окрестности анормальной точки, Матем. заметкu, 2005, том 77, выпуск 4, 483-497

DOI: https://doi.org/10.4213/mzm2507

Использование Общероссийского математического портала Math-Net.Ru подразумевает, что вы прочитали и согласны с пользовательским соглашением http://www.mathnet.ru/rus/agreement

Параметры загрузки:

IP : 54.237 .59 .107

26 апреля 2023 г., $13: 33: 12$

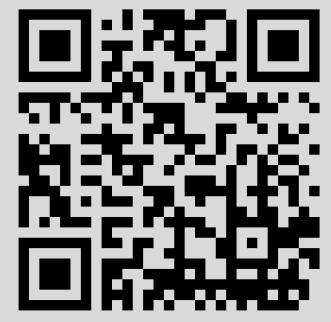




\section{НАКРЫВАНИЕ НЕЛИНЕЙНЫХ ОТОБРАЖЕНИЙ НА КОНУСЕ В ОКРЕСТНОСТИ АНОРМАЛЬНОЙ ТОЧКИ}

\section{А. В. Арутюнов}

Работа посвящена теоремам об обратной функции для гладких нелинейных отображений, заданных на выпуклом конусе банахова пространства в окрестности анормальной точки. Доказана соответствующая теорема о накрывании. Вывод этих теорем основан на полученной теореме Банаха об открытом отображении для выпуклого конуса банахова пространства. Получены достаточные условия касания к множеству нулей нелинейного отображения без априорных предположений нормальности.

Библиографоия: 13 названий.

1. Введение. Пусть $X$ и $Y$-банаховы пространства, $K \subseteq X$-выпукльй замкнутый конус, $F: X \rightarrow Y$ - отображение, которое дважды непрерывно дифференцируемо по $\Phi$ реше [1] в окрестности заданной точки $x_{0} \in X$. Пусть $y_{0}=F\left(x_{0}\right)$ и $\varphi:[0,1] \rightarrow Y-$ непрерьвная кривая в $Y$, начинающаяся в точке $y_{0}: \varphi(0)=y_{0}$. Пусть также в $Y$ задано семейство подмножеств $\Gamma(r)$, зависящее от скалярного параметра $r \geqslant 0$, причем это семейство возрастает по включению, т.е. $\Gamma\left(r_{1}\right) \subset \Gamma\left(r_{2}\right) \forall r_{1}<r_{2}$, и $\Gamma(0)=\{0\}$.

Обозначим через $B_{r}(x)=\{\xi \in X:\|\xi-x\| \leqslant r\}$ замкнутый шар в $X$ радиуса $r$ с центром в точке $x$. Аналогичньй шар в $Y$ также будем обозначать через $B_{r}(y)$. Положим $B_{r}=B_{r}(0)$.

ОПРЕДЕЛЕНИЕ. Отображение $F$ Г-накрывает в точке $x_{0}$ по кривой $\varphi$ относительно конуса $K$, если существует число $r_{0}>0$ такое, что для любого $r \in\left[0, r_{0}\right]$ выполняется

$$
\varphi(r)+\Gamma(r) \subseteq F\left(B_{r}\left(x_{0}\right) \cap\left(x_{0}+K\right)\right) .
$$

Если $\varphi(r) \equiv y_{0}$, то будем говорить, что $F$ Г-накрывает в точке $x_{0}$ относительно $K$ (опуская словосочетание “по кривой $\varphi$ ”). Мы изучим критерии линейно-квадратичных Г-накрываний в точке, т.е. когда

$$
\Gamma(r)=\left(B_{b r} \cap \widetilde{C}\right)+B_{b r^{2}},
$$

где $\widetilde{C}$ - заданньй замкнутый вьпукльй конус из $Y$ и $b>0$. Условия Г-накрьвания интересны не только сами по себе. Они имеют различные приложения (теория экстремальных задач, управляемость, устойчивость решений уравнений и их зависимость от начальных данных и т.д.).

Работа выполнена при поддержке Российского фонда фундаментальных исследований, грант № 05-01-00193. 
Наряду с Г-накрьванием определим еще один вид накрывания, который назовем линейньм. А именно, скажем, что отображение $F$ линейно накрывает в окрестности $V$ точки $x_{0}$ относительно конуса $K$ с коэффициентом $b$, если

$$
\exists r_{0}>0: F(x)+B_{b r} \subseteq F\left(B_{r}(x) \cap\left(x_{0}+K\right)\right) \quad \forall r \in\left[0, r_{0}\right], \quad \forall x \in V \cap\left(x_{0}+K\right) .
$$

Принципиальное отличие линейного накрьвания от Г-накрьвания заключается в том, что в (1) участвуют окрестности только одной точки $x_{0}$, а в линейном накрьвании окрестности всех точек $x \in V \cap\left(x_{0}+K\right)$.

Наиболее изучен случай линейного накрьвания. Линейное накрьвание в окрестности точки $x_{0}$ относительно конуса $K$ гарантируется вьполнением условия Робинсона $F^{\prime}\left(x_{0}\right)(K)=Y$ (это обобщение условия Люстерника $F^{\prime}\left(x_{0}\right)(X)=Y$ (см. [1]) на случай конусов). Действительно, из условия Робинсона вытекает (см. [2]) существование такого $a>0$, что $\forall y \in Y \quad \exists x \in K: F^{\prime}\left(x_{0}\right) x=y,\|x\| \leqslant a\|y\|$. Для доказательства линейного накрьвания исходного нелинейного отображения $F$ в окрестности точки $x_{0}$ осталось к линейно накрьвающему линейному оператору $F^{\prime}\left(x_{0}\right)$ применить теорему Милютина [3, теорема 1.3], гарантирующую, что если к линейно накрывающему отображению с коэффициентом $b$ прибавить любое отображение с константой Липшища $c<b$, то их сумма будет линейно накрывающим отображением с коэффициентом $b-c$.

Если условие Робинсона нарушается (в этом случае точка $x_{0}$ назьвается анормальной), то линейное накрьвание, вообще говоря, невозможно. Е. Р. Аваковьп в [4] доказан следующий результат: пусть $K=X$, образ первой производной $\operatorname{Im} F^{\prime}\left(x_{0}\right)$ является замкнутым топологически дополняемым подпространством и ${ }^{1} \exists h \in X: F^{\prime}\left(x_{0}\right) h=0$, $F^{\prime \prime}\left(x_{0}\right)[h, h] \in \operatorname{Im} F^{\prime}\left(x_{0}\right), \operatorname{Im} F^{\prime}\left(x_{0}\right)+F^{\prime \prime}\left(x_{0}\right)\left[h, \operatorname{Ker} F^{\prime}\left(x_{0}\right)\right]=Y$. Тогда отображение $F$ $\Gamma$-накрывает в точке $x_{0}$, где семейство $\Gamma(r)$ определяется в силу (2) при $\widetilde{C}=\operatorname{Im} F^{\prime}\left(x_{0}\right)$.

В настоящей работе этот результат обобщается на случай конуса, т.е. когда $K \neq X$. Предлагаемьй вьвод теоремы о накрьвании относительно конуса $K$ основан на описанном вьше подходе, использующем цитированную теорему Милютина о возмущении линейно накрьвающего отображения. Для его реализации вначале докажем конусную теорему Банаха.

2. Теорема Банаха для конуса. Пусть $X$ и $Y$ - банаховы пространства, $K \subseteq X$ выпукльй замкнутьй конус, $A: X \rightarrow Y$ - линейный ограниченньй оператор. Положим $C=A(K)$. Обозначим через $\operatorname{Lin} C$ линейную оболочку конуса $C$, а через $\operatorname{ri} C$ относительную внутренность $C$. Будем предполагать, что подпространство Lin $C$ замкнуто и ri $C \neq \varnothing$. (Эти предположения автоматически вьполняются, если $Y$ конечномерно.) Спрашивается, верно ли, что

$$
\exists a>0: \quad \forall y \in C \quad \exists x \in K: A x=y, \quad\|x\| \leqslant a\|y\| ?
$$

Иными словами, справедливо ли конусное обобщение теоремы Банаха об открытом отображении? Если не предполагать замкнутость конуса $C$, то соответствующий контрпример строится тривиально. Поэтому предположим, что конус $C$ замкнут. Если, кроме того, конус $C$ конечнопорожденный, т.е. является выпуклой оболочкой конечного

\footnotetext{
${ }^{1}$ Здесь и ниже для произвольного подмножества $\widetilde{X}$ запись $F^{\prime \prime}\left(x_{0}\right)[h, \widetilde{X}]$ обозначает множество, полученное объединением векторов $F^{\prime \prime}\left(x_{0}\right)[h, \xi]$ по всем $\xi \in \widetilde{X}$.
} 
числа лучей, то существование искомого $a$ доказывается несложно ${ }^{2}$. В общем же случае даже в предположении замкнутости и конечномерности $C$ ответ на поставленньй вопрос отрицателен, что показьвает следующий

ПримеР 1. Пусть $X=l_{2}, K=\left(x^{1}, x^{2}, \ldots\right) \in l_{2}: x^{i} \geqslant 0 \forall i$ - неотрищательньй ортант в $l_{2}, Y=\mathbb{R}^{3}$. Выберем в $\mathbb{R}^{3}$ последовательность векторов $d_{i}$ следующим образом: $d_{i}=\left(q_{i}, 1\right)$. Здесь $q_{i}$ - двумерный единичный вектор из $\mathbb{R}^{2}$, составляющий с осью абсцисс угол $2 \pi\left(1-(i+1)^{-1}\right), i=0,1,2, \ldots$ Тогда $\left|d_{i}\right|=\sqrt{2} \forall i, d_{i} \rightarrow d_{0}$.

Определим линейньй непрерьвный оператор $A$ на единичньх ортах $e_{i}=(0, \ldots, 0,1$, $0, \ldots$ ) (единица на $i$-м месте) по формуле $A e_{i}=i^{-1} d_{i}$, т.е. $A x=\sum_{i=1}^{\infty} i^{-1} x^{i} d_{i}$. Через $C$ обозначим выпуклую коническую оболочку векторов $d_{i}, i=0,1, \ldots$.

Непосредственно проверяется, что $A(K)=C$, конус $C$ замкнут и в каждый вектор $d_{i}$ оператором $A$ переводится единственный вектор из $K$ - это вектор $i e_{i}$. Следовательно, (3) не вьполняется.

Отметим, что пример 1 можно модифицировать (эту идею предложил М. Ф. Сухинин) так, что (3) нарушается уже при $X=\mathbb{R}^{4}$. В то же время справедлива следующая

ТЕОрема 1. Для любого $y_{0} \in \operatorname{ri} C$ существуют такие (зависящие от $\left.y_{0}\right) \quad \delta>0$ $u a>0$, чmo

$$
\forall y \in \text { cone } B_{\delta}\left(y_{0}\right) \cap C \quad \exists x \in K: A x=y, \quad\|x\| \leqslant a\|y\| .
$$

Здесь cone $B=\bigcup_{\alpha \geqslant 0} \alpha B-$ коническая оболочка мнохества $B$.

ДокАЗАТЕЛьСтво. Эта теорема может быть вьведена из теоремы 1 из [5, с. 136], однако мы приведем прямое доказательство. Для произвольного $y \in C$ рассмотрим задачу

$$
\|x\| \rightarrow \inf , \quad A x=y, \quad x \in K
$$

$(y$ - параметр). Через $p(y)$ обозначим инфимум в ней. По построению $p(y)<\infty \forall y \in C$ и, кроме того, функция $p$, очевидно, вьпукла. Докажем, что $p$ ограничена сверху на некотором не пустом, открытом относительно $\operatorname{Lin} C$ подмножестве. Для этого, вначале, докажем утверждение, являющееся модификацией леммы из [6, с. 260].

Лемма 1. Пусть $B-$ замкнутый шар, лежаший в $\operatorname{int} C, u \mathscr{B}=\bigcup_{\alpha \in(0,1]} \alpha B-$ объединение множеств $\alpha B$ по всем $\alpha \in(0,1]$. Пусть множсество $D$ всюду плотно в $\mathscr{B}$. Тогда произвольный $y \in \operatorname{int} B, y \neq 0$, можно разложить в ряд так, что

$$
y=\sum_{i=1}^{\infty} y_{i}, \quad y_{i} \in D, \quad\left\|y_{i}\right\| \leqslant 3\|y\| 2^{-i} .
$$

${ }^{2}$ Действительно, если конус $C$ конечнопорожденньй, то, не теряя общности, можно считать, что $Y=\mathbb{R}^{n}$, a $\mathbb{R}^{n}$ представимо в виде конечного объединения острых (т.е. не содержащих ненулевых подпространств) конечнопорожденных конусов $Y_{i}$. (В качестве $Y_{i}$ можно взять всевозможные ортанты). Каждый из конусов $C_{i}=C \cap Y_{i}$ также является острым и по теореме $\Gamma$. Вейля конечнопорожденньм. Пусть $I$ это множество индексов $i$, для которых $C_{i} \neq\{0\}$. Пусть $i \in I$, a $e_{i, 1}, \ldots, e_{i, l}-$ все единичные ребра конуса $C_{i}$. В силу его остроты $\exists \delta_{i}>0, y_{i}:\left\langle y_{i}, e_{i, j}\right\rangle>\delta_{i} \forall j,\left|y_{i}\right|=1$. Выберем $x_{i, j} \in K: A x_{i, j}=e_{i, j}$ и $\alpha:\left\|x_{i, j}\right\| \leqslant \alpha \forall i, j$. Пусть $y \in Y_{i}$. Тогда $y=\sum_{j} \lambda_{j} e_{i, j}$, где $\lambda_{j} \geqslant 0$. Умножая это равенство скалярно на $y_{i}$, имеем $\sum_{j} \lambda_{j} \leqslant|y| / \delta_{i}$. Положим $x=\sum_{j} \lambda_{j} x_{i, j}$. Тогда $A x=y,\|x\| \leqslant \alpha \sum_{j} \lambda_{j} \leqslant|y| \alpha / \delta_{i}$. Поэтому для $a=\alpha \max _{i \in I} \delta_{i}^{-1}$ имеет место (3). 
ДокАЗАТЕЛЬСтво. Покажем, что для любого $\eta \in \operatorname{int} \mathscr{B}, \eta \neq 0$, вьполняется

$$
\exists \xi \in D: 0 \neq \eta-\xi \in \operatorname{int} \mathscr{B}, \quad\|\eta-\xi\| \leqslant \frac{1}{2}\|\eta\| .
$$

Очевидно, $\eta-\frac{3}{4} \eta=\frac{1}{4} \eta \in \operatorname{int} \mathscr{B}$. Поэтому в $D$ найдется такой достаточно близкий к $\frac{3}{4} \eta$ вектор $\xi \in D$, что $0 \neq \eta-\xi \in$ int $\mathscr{B},\left\|\frac{3}{4} \eta-\xi\right\| \leqslant \frac{1}{4}\|\eta\|$. Очевидно,

$$
\|\eta-\xi\| \leqslant \frac{1}{4}\|\eta\|+\left\|\frac{3}{4} \eta-\xi\right\| \leqslant \frac{1}{4}\|\eta\|+\frac{1}{4}\|\eta\|=\frac{1}{2}\|\eta\|
$$

и, значит, для $\xi$ выполняется (6).

Возьмем произвольньй $y \in B, y \neq 0$, и для него построим искомое разложение. Положим $\eta=y$. В силу (6) существует такой $y_{1}=\xi$, что

$$
y_{1} \in D, \quad 0 \neq y-y_{1} \in \operatorname{int} \mathscr{B}, \quad\left\|y-y_{1}\right\| \leqslant 2^{-1}\|y\| .
$$

Далее, взяв в (6) $\eta=y-y_{1}$, выберем $y_{2}=\xi \in D$ так, что

$$
0 \neq y-y_{1}-y_{2} \text { int } \mathscr{B}, \quad\left\|y-y_{1}-y_{2}\right\| \leqslant 2^{-1}\left\|y-y_{1}\right\| \leqslant 2^{-2}\|y\| .
$$

И вообще, построив $y_{1}, \ldots, y_{i-1}$, положим $\eta=y-\sum_{l=1}^{i-1} y_{l}$. В силу (6) выберем $y_{i}=$ $\xi \in D$ так, что

$$
0 \neq y-\sum_{l=1}^{i} y_{l} \text { int } \mathscr{B}, \quad\left\|y-\sum_{l=1}^{i} y_{l}\right\| \leqslant \frac{1}{2}\left\|y-\sum_{l=1}^{i-1} y_{l}\right\| \leqslant \frac{1}{2} \cdot 2^{-(i-1)}\|y\|=2^{-i}\|y\| .
$$

Процесс построения последовательности $\left\{y_{i}\right\}$ описан. По построению имеем $\| y-$ $\sum_{l=1}^{i} y_{l} \| \rightarrow 0, i \rightarrow \infty$, т.е. ряд $\sum_{i} y_{i}$ сходится. Оценим нормы $y_{i}$. Имеем $\left\|y_{1}\right\| \leqslant\left\|y-y_{1}\right\|$ $+\|y\| \leqslant 2^{-1} 3\|y\|$

$$
\left\|y_{i}\right\| \leqslant\left\|\sum_{l=1}^{i} y_{l}-y\right\|+\left\|y-\sum_{l=1}^{i-1}\right\| \leqslant 2^{-i}\|y\|+2^{-(i-1)}\|y\|=2^{-i} 3\|y\|,
$$

т.е. это разложение является искомым. Лемма доказана.

Вернемся к доказательству ограниченности функции $p$. Для натуральных $i$ положим $C_{i}=\{y: y=A x, x \in K,\|x\| \leqslant i\|y\|\}$. Тогда $\cup_{i} C_{i}=C$. Рассмотрим банахово пространство Lin $C$. По теореме Бэра существует такое натуральное $N$, что $C_{N}$ всюду плотно в некотором замкнутом шаре $B$ пространства $\operatorname{Lin} C$, принадлежащем ri $C$ (ведь ri $C \neq \varnothing)$. Положим $D=\bigcup_{\alpha \in(0,1]} \alpha\left(C_{N} \cap B\right)$. Очевидно, $D$ всюду плотно в $\mathscr{B}=\cup_{\alpha \in(0,1]} \alpha B$.

Возьмем произвольное $y \in$ ri $B, y \neq 0$. По лемме 1 существует разложение (5). По построению $y_{i} \in D \Longrightarrow \exists x_{i} \in K: A x_{i}=y_{i},\left\|x_{i}\right\| \leqslant N\left\|y_{i}\right\| ;\left\|y_{i}\right\| \leqslant 3\|y\| 2^{-i} \Longrightarrow\left\|x_{i}\right\| \leqslant$ $3 N\|y\| 2^{-i} \forall i$. Поэтому ряд $\sum_{i=1}^{\infty} x_{i}$ сходится к некоторому $x$, причем $x \in K$ в силу замкнутости $K$. Применяя почленно к ряду $x=\sum_{i=1}^{\infty} x_{i}$ непрерывный оператор $A$, получаем $A x=\sum_{i=1}^{\infty} y_{i}=y$. Кроме того, $\|x\| \leqslant \sum_{i=1}^{\infty}\left\|x_{i}\right\| \leqslant 3 N\|y\|$. Таким образом, $\forall y \in \operatorname{ri} B \quad \exists x \in K: A x=y,\|x\| \leqslant 3 N\|y\|$, и, значит, на шаре $\operatorname{ri} B$ функция $p$ ограничена сверху числом $3 N$ (ведь $p(0)=0$ ). Таким образом, на конусе $C$ функция $p$ конечна, выпукла и ограничена сверху на не пустом относительно открытом подмножестве. Поэтому $p$ непрерьвна на ri $C$ и, значит, для каждой точки $y_{0} \in \operatorname{ri} C$ она ограничена в ее относительной окрестности. Отсюда непосредственно вытекает справедливость (4) для некоторых $a>0, \delta>0$. Теорема доказана.

Приведем следствия теоремы 1. 
СлЕДСТВИЕ 1. Пусть конус $C$ содержит замкнутое подпространство $Y_{1}$. Тогда $\exists a>0: \forall y \in Y_{1} \quad \exists x \in K: A x=y,\|x\| \leqslant a\|y\|$. Таким образом, классическая теорема Банаха об открытом отобрахсении формально следует из теоремы 1.

СледСтвие 2. Пусть $x_{0} \in K: A x_{0}=y_{0} \in \operatorname{ri} C$. Тогда существуют такие (зависящие от $\left.x_{0}\right) \quad \delta>0$ и а >

$\forall \xi \in B_{\delta}\left(x_{0}\right) \cap K \quad \forall y \in B_{\delta}\left(y_{0}\right) \cap \operatorname{Lin} C \quad \exists x \in K: A x=y, \quad\|x-\xi\| \leqslant a\|y-A \xi\|$.

ДокАЗАТЕЛЬСТво. В силу замкнутости $\operatorname{Lin} C$, не теряя общности, будем считать, что $Y=\operatorname{Lin} C \Longrightarrow y_{0} \in \operatorname{int} C$. Возьмем $\delta>0$, отвечающее $y_{0}$ в силу теоремы 1 . Выберем $\varepsilon>0$ так, что $A\left(B_{\varepsilon}\left(x_{0}\right)\right) \subseteq B_{\delta}\left(y_{0}\right)$. Для произвольных фиксированных $y \in B_{\delta}\left(y_{0}\right)$, $\xi \in B_{\varepsilon}\left(x_{0}\right)$ рассмотрим задачу минимизации по переменной $x$

$$
\|x-\xi\| \rightarrow \inf , \quad A x=y, \quad x \in K
$$

$\left(\xi, y\right.$ - параметры). Инфимум в ней обозначим через $p_{\xi}(y)$. Каждая из функций $p_{\xi}$ выпукла и в силу теоремы 1 эти функции $p_{\xi}$ ограничены равномерно по $\xi \in B_{\varepsilon}\left(x_{0}\right)$ на открытом множестве $B_{\delta}\left(y_{0}\right)$. Поэтому (см. $[7$, с. 22,23$\left.]\right)$ в $(\delta / 2)$-окрестности $y_{0}$ все функции $p_{\xi}$ удовлетворяют условию Липшица с одной и той же константой Липшица $a$. Из очевидных соотношений $p_{\xi}(A \xi)=0 \forall \xi \in B_{\varepsilon}\left(x_{0}\right) \cap K \Longrightarrow p_{\xi}(y)=$ $\left|p_{\xi}(y)-p_{\xi}(A \xi)\right| \leqslant a\|y-A \xi\|$ получаем требуемое. Следствие доказано.

СлЕДСТВИЕ 3. Пусть пространство $Y$ конечномерно. Тогда для любого замкнутого выпуклого конуса $\widetilde{C}$ такого, что $\widetilde{C} \backslash\{0\} \subset \operatorname{ri} C$, выполняется

$$
\exists a>0: \forall y \in \widetilde{C} \quad \exists x \in K: A x=y, \quad\|x\| \leqslant a\|y\| .
$$

Это следствие теоремы 1 и компактности единичной сфреры конечномерного пространства.

Следуюший пример показывает, что в следствии 3 предположение о конечномерности $Y$ существенно.

ПРИмеР 2. Суть конструкции такова. Пусть $Y$ - бесконечномерноегильбертово пространство, а $\widetilde{C}$ и $C$-ненулевые замкнутыевьпуклые конусы в нем, причем $B_{\delta}(\widetilde{C} \cap S) \subset C$ для некоторого $\delta>0$. Здесь $S$ - единичная сфера, а $B_{\delta}(M)-\delta$-окрестность множества $M$. Предположим, что существует определенная на $C$ конечная неотрицательная положительно-однородная выпуклая функция $p$, которая непрерьвна на $C \backslash\{0\}$, однако неограничена на $\widetilde{C} \cap S$. Доопределив функцию $p$ вне $C$ как $+\infty$, рассмотрим в гильбертовом пространстве $X=Y \times \mathbb{R}^{1}$ ее надграфик $K=\{x: x=(y, \alpha), y \in C, \alpha \geqslant p(y)\}$. Очевидно, $K$ - выпуклый замкнутый конус. Определим линейный непрерьвньй оператор проектирования $A: X \rightarrow Y$ формулой $A x=y, x=\left(y, y^{0}\right), y \in Y, y^{0} \in \mathbb{R}^{1}$. Тогда $A(K)=C$. В то же время для конуса $\widetilde{C}$ условие (7) нарушается, так как по построению $|x| \geqslant p(y) \forall y \in C \forall x \in K: A x=y$, а по предположению функция $p$ неограничена на $\widetilde{C} \cap S$. Нам осталось построить искомые конусы $C, \widetilde{C}$ и функцию $p$. Сделаем это.

Итак, пусть $Y$ - гильбертово пространство с ортонормированным базисом $e_{0}, e_{1}, \ldots$ Конус $C$ определим так:

$$
C=\left\{y: y=\alpha\left(e_{0}+e\right), \quad \alpha \geqslant 0, \quad e \in Y,\left\langle e, e_{0}\right\rangle=0, \quad|e| \leqslant 1\right\} .
$$


Очевидно, конус $C$ выпукльй и замкнутый. Выберем число $\beta \in(-1,0)$ так, что $2 \beta^{2}>1$. Положим $g_{n}=\beta e_{0}+e_{n}, G_{n}=\left\{y \in Y:\left\langle y, g_{n}\right\rangle \geqslant 0\right\}, n=1,2, \ldots$ Используя определение $\beta$, непосредственной проверкой получаем, что $C \cap G_{i} \cap G_{j}=\{0\}$ $\forall i \neq j$. Для $y \in C$ положим $p(y)=\max _{n \geqslant 0}\left(n\left\langle g_{n}, y\right\rangle\right)$, где $g_{0}=0$. Функция $p$ определена корректно и конечна на $C$, так как в силу сказанного вьше для любого $y \in C$ неравенство $\left\langle g_{n}, y\right\rangle>0$ выполняется не более, чем для одного натурального $n$. Ясно, что функция $p$ положительно-однородна, вьпукла и непрерывна на $C$ в каждой точке $y \neq 0$.

Выберем $\gamma<1$ так, что $\beta+\gamma>0$, и определим конус $\widetilde{C}=\left\{y: y=\alpha\left(e_{0}+e\right), \alpha \geqslant 0\right.$, $\left.e \in Y,\left\langle e, e_{0}\right\rangle=0,|e| \leqslant \gamma\right\}$. Легко видеть, что $B_{\delta}(\widetilde{C} \cap S) \subset C$ для $\delta=\frac{1}{2}(1-\gamma)\left(1+\gamma^{2}\right)^{-1 / 2}$. В то же время для векторов $y_{n}=\left(1+\gamma^{2}\right)^{-1 / 2}\left({\underset{e}{0}}_{\widetilde{C}}+\gamma e_{n}\right)$ имеем $y_{n} \in \widetilde{C} \cap S, p\left(y_{n}\right)=$ $n\left(1+\gamma^{2}\right)^{-1 / 2}(\beta+\gamma) \rightarrow \infty, n \rightarrow \infty$. Искомые $C, \widetilde{C}, p$ построены.

Следуюший результат понадобится ниже.

ПРЕДЛОЖЕНИЕ 1. Пусть линейный непрерывный оператор $A: X \rightarrow Y$ линейно накрывает в некоторой окрестности $V$ точки $x_{0} \in K$ относительно конуса $K$ $c$ коэффициентом $b>0$. Тогда существует $r_{0}>0$ такое, что $\forall r \in\left(0, r_{0}\right] u$ для любого отображения $f: V \rightarrow Y$, для которого $\left\|f\left(x_{0}\right)\right\|<b r / 2$ и выполнено условие Липшица с константой $b / 2$, уравнение $A x=A x_{0}+f(x)$ имеет решение $x \in K \cap B_{r}\left(x_{0}\right)$.

ДокАЗАтЕльство. Определим отображение $F$ по формуле $F(x)=A x-A x_{0}-$ $f(x)+f\left(x_{0}\right)$. По теореме Милютина (теорема 1.3 из [3]) отображение $F$ линейно накрывает в окрестности $x_{0}$ относительно конуса $K$ с коэффициентом $b / 2$. Поэтому, так как $F\left(x_{0}\right)=0$, то существует $r_{0}>0$, не зависящее от $f$, такое, что $F\left(K \cap B_{r}\left(x_{0}\right)\right) \supseteq B_{b r / 2}$ $\forall r \in\left(0, r_{0}\right]$. Следовательно, эквивалентное исходному уравнение $F(x)=f\left(x_{0}\right)$ имеет решение $x \in K \cap B_{r}\left(x_{0}\right)$. Предложение доказано.

3. Теорема о накрывании. Далее предполагается, что в окрестности точки $x_{0}$ отображение $F: X \rightarrow Y$ дважды непрерьвно дифференцируемо по Фреше и $F^{\prime \prime}(\cdot)$ липшищево. Положим $C=F^{\prime}\left(x_{0}\right)(K)$. Также предполагается, что подпространство $Y_{1}=$ Lin $C$ замкнуто и ri $C$ не пусто. (Эти предположения автоматически вьполняются, если $Y$ конечномерно.)

ОПРЕДЕЛЕНИЕ. Пусть $h \in K$. Скажем, что отображение $F$ 2-регулярно в точке $x_{0}$ относительно $K$ по направлению $h$, если

$$
Y_{1}+F^{\prime \prime}\left(x_{0}\right)\left[h, \operatorname{Ker} F^{\prime}\left(x_{0}\right) \cap K\right]=Y .
$$

Теорема 2. Предположим, что $h \in K$ и отображсение $F$ 2-регулярно в точке $x_{0}$ относительно конуса $K$ по направлению $h$, причем $\|h\|<1$. Тогда для любых векторов $l, m \in \mathrm{ri} C$ найдутся такие $\delta>0, b>0$, что отображсение $F$ Г-накрывает в точке $x_{0}$ по кривой $\varphi$ относительно конуса $K$. Здесь

$$
\varphi(r)=y_{0}+r F^{\prime}\left(x_{0}\right) h+r^{2}\left(\frac{1}{2} F^{\prime \prime}\left(x_{0}\right)[h, h]+m\right),
$$

а Г определяется по формуле (2) при $\widetilde{C}=$ cone $B_{\delta}(l) \cap Y_{1}$. 
ДокАЗАТЕЛЬСтво. Важное место играет следующее простое соображение: если вектор $\bar{y}$ лежит в относительной внутренности выпуклого конуса $C$, то произвольный вектор $y \in \operatorname{Lin} C$ представим в виде $y=\tilde{y}-\alpha \bar{y}$ для некоторых $\tilde{y} \in C, \alpha \geqslant 0$. Перейдем к строгим рассуждениям.

Пусть для удобства $F\left(x_{0}\right)=0, x_{0}=0$. Положим $Q=\frac{1}{2} F^{\prime \prime}\left(x_{0}\right)$. По лемме Адамара в окрестности $x_{0}$ справедливо представление: $F(x)=F^{\prime}\left(x_{0}\right) x+Q[x, x]+R(x)[x, x]$, где при каждом фиксированном $\xi R(\xi)$ - квадратичное отображение, причем $R(0)=0$ и $R(\cdot)$ липшицево в окрестности нуля.

По условию $l \in \mathrm{ri} C$. Поэтому по теореме 1 существуют такие $a_{1}>0, \delta>0$, что для $\widetilde{C}=$ cone $B_{\delta}(l) \cap Y_{1}$ выполняется

$$
\forall \tilde{y} \in \widetilde{C} \quad \exists \chi(\tilde{y}) \in K: F^{\prime}\left(x_{0}\right) \chi(\tilde{y})=\tilde{y}, \quad\|\chi(\tilde{y})\| \leqslant a_{1}\|\tilde{y}\| .
$$

Возьмем $\tilde{y} \in \widetilde{C}, y \in Y$ и для $\varepsilon>0$ будем решать уравнение

$$
F(x)=\varepsilon\left(F^{\prime}\left(x_{0}\right) h+\tilde{y}\right)+\varepsilon^{2}(Q[h, h]+m+y), \quad x \in K .
$$

Его решение будем искать в виде $x=x(\varepsilon)=\varepsilon \hat{x}\left(\varepsilon, x_{1}, x_{2}, \tilde{y}\right)$, где $\hat{x}\left(\varepsilon, x_{1}, x_{2}, \tilde{y}\right)=h+$ $\chi(\tilde{y})+\varepsilon x_{1}+x_{2}, x_{1} \in K, x_{2} \in K_{2}$. Здесь $K_{2}=K \cap \operatorname{Ker} L_{1}, L_{1}=F^{\prime}\left(x_{0}\right), L_{1}: K \rightarrow Y_{1}$.

Очевидно, $x(\varepsilon) \in K$. Имеем

$$
F\left(\varepsilon h+\varepsilon \chi(\tilde{y})+\varepsilon^{2} x_{1}+\varepsilon x_{2}\right)=\varepsilon\left(L_{1} h+\tilde{y}\right)+\varepsilon^{2}(Q[h, h]+m+y) .
$$

Возьмем $\bar{x}_{1} \in K: L_{1} \bar{x}_{1}=m$. Нам достаточно доказать существование таких $r_{1}, r_{2}$, $\varepsilon_{0}>0$, что для любых $y \in Y, \tilde{y} \in \widetilde{C}:\|y\| \leqslant r_{2},\|\tilde{y}\| \leqslant r_{2}, \varepsilon \in\left(0, \varepsilon_{0}\right)$ уравнение (12) имеет решение $x_{1} \in K, x_{2} \in K_{2}$, для которого

$$
\left\|x_{1}-\bar{x}_{1}\right\| \leqslant r_{1}, \quad\left\|x_{2}\right\| \leqslant r_{1}, \quad\left\|\hat{x}\left(\varepsilon, x_{1}, x_{2}, \tilde{y}\right)\right\|<1 .
$$

Удовлетворяющие этим неравенствам $x_{1}, x_{2}, y, \tilde{y}, \varepsilon$ назовем допустимыми.

По условию $\|h\|<1$. Поэтому найдутся такие положительные $r_{1}, r_{2}, \varepsilon_{0}$, что $a_{1} r_{2}+$ $\varepsilon_{0}\left(\|m\|+r_{1}\right)+r_{2}<1-\|h\|$. По условию $\bar{x}_{1} \in K, L_{1} \bar{x}_{1}=m \in \operatorname{ri} C$. Поэтому в силу следствия 2 к теореме 1 сужение отображения $L_{1}$ на $K$ линейно накрывает в пересечении $K$ с окрестностью $\bar{x}_{1}$. Определим линейный оператор $L_{2}: K_{2} \rightarrow Y$ формулой $L_{2} x_{2}=2 Q\left[h, x_{2}\right]$. Рассмотрим банахово факторпространство $Y / Y_{1}$ и факторотображение $\pi: Y \rightarrow Y / Y_{1}$. В силу (9) $\pi L_{2}\left(K_{2}\right)=Y / Y_{1}$ и, значит, в силу следствия 1 к теореме 1 оператор $\pi \circ L_{2}$ линейно накрывает на $K_{2}$. Из сказанного несложно получаем, что линейньй оператор $A: K \times K_{2} \rightarrow Y$, определенный по формуле $A\left(x_{1}, x_{2}\right)=L_{1} x_{1}+L_{2} x_{2}$, линейно накрывает в некоторой окрестности точки $\left(\bar{x}_{1}, 0\right)$.

Исследуем уравнение (12). Подставляя в (12) представление для $F$, вычитая из обеих частей равенства $\varepsilon L_{1} h+\varepsilon^{2} Q[h, h]$ и учитывая $(11)$, после деления на $\varepsilon^{2}$ получаем

$$
A\left(x_{1}, x_{2}\right)=m+f\left(x_{1}, x_{2} ; \tilde{y}, y, \varepsilon\right) .
$$

Здесь

$$
\begin{aligned}
f\left(x_{1}, x_{2} ; \tilde{y}, y, \varepsilon\right)= & y-2 Q\left[h, \varepsilon x_{1}\right]-Q\left[\varepsilon x_{1}+x_{2}\right]^{2} \\
& -Q\left[2\left(h+\varepsilon x_{1}+x_{2}\right)+\chi(\tilde{y}), \chi(\tilde{y})\right]-R(x(\varepsilon))\left[h+\chi(\tilde{y})+\varepsilon x_{1}+x_{2}\right]^{2}
\end{aligned}
$$


и использовано обозначение $Q[x]^{2}=Q[x, x]$.

В окрестности точки $\left(\bar{x}_{1}, 0\right)$ отображение $A$ линейно накрывает с некоторым коэффициентом $b>0$. Уменьшая положительные $r_{1}, r_{2}$ и $\varepsilon_{0}$, в силу $(11)$, используя явный вид функции $f$, добьемся того, что для любых допустимых $x_{1}, x_{2}, \tilde{y}, y, \varepsilon$ выполняется $\left\|f\left(\bar{x}_{1}, 0 ; \tilde{y}, y, \varepsilon\right)\right\|<b r_{1} / 2$ и при этом константа Липшица функций $f(\cdot ; \tilde{y}, y, \varepsilon)$ по переменным $x_{1}, x_{2}$ на множестве $\mathscr{X}=\left\{x_{1} \in K, x_{2} \in K_{2}:\left\|x_{1}-\bar{x}_{1}\right\|+\left\|x_{2}\right\| \leqslant r_{1}\right\}$ не превышает $b / 2$.

Таким образом, так как $m=A\left(\bar{x}_{1}, 0\right)$, для уравнения $(13)$ в точке $\left(\bar{x}_{1}, 0\right)$ вьполнены все условия предложения 1. Из него вытекает, что для любых допустимых $\tilde{y}, y, \varepsilon$ уравнение (13) имеет решение $x_{1}, x_{2} \in \mathscr{X}$. При этом по построению $\left\|\hat{x}\left(\varepsilon, x_{1}, x_{2}, \tilde{y}\right)\right\|<1$. Теорема доказана.

Основной частньй, но наиболее употребительный случай теоремы 2 - это когда вектор $h \in K$ удовлетворяет условиям

$$
F^{\prime}\left(x_{0}\right) h=0, \quad\left(-F^{\prime \prime}\left(x_{0}\right)[h, h]\right) \in \operatorname{ri} C .
$$

Тогда берем $m=-\frac{1}{2} F^{\prime \prime}\left(x_{0}\right)[h, h]$, откуда $\varphi(r) \equiv y_{0}$ и мы получаем $\Gamma$-накрывание в точке $x_{0}$ вдоль конуса $K$.

Пусть вьполняется (14). Тогда из (9) вытекает, что

$$
F^{\prime}\left(x_{0}\right)(K)+F^{\prime \prime}\left(x_{0}\right)\left[h, K \cap \operatorname{Ker} F^{\prime}\left(x_{0}\right)\right]=Y
$$

и, значит, это условие, предложенное Е.Р. Аваковьм, эквивалентно (9). Действительно, пусть имеет место (9). Возьмем $y \in Y$. В силу (9) $\exists y_{1} \in Y_{1}, \tilde{x} \in \operatorname{Ker} L_{1} \cap K: y=$ $y_{1}+2 Q[h, \tilde{x}]$. (Мы используем обозначения, введенные при доказательстве теоремы 2.) Но так как $m=-2 Q[h, h]$ лежит в относительной внутренности конуса $C$, то вектор $y_{1} \in \operatorname{Lin} C$ представим в виде $y_{1}=c-\alpha m$ для некоторых $c \in C, \alpha \geqslant 0$. Поэтому $y_{1}=c+2 Q[h, \alpha h]$. Выберем $x_{1} \in K: L_{1} x_{1}=c$ и положим $x_{2}=\tilde{x}+\alpha h$. Очевидно, $y=A\left(x_{1}, x_{2}\right)$. Равенство $A\left(K \times K_{2}\right)=Y$ доказано. При $K=X$ условия $\Gamma$-накрывания в смысле теоремы 2 получены в [8], а также при иных предположениях в [9].

Теорема 2'. Пусть пространство Y конечномерно. Предположим, что $h \in K$ $u$ отображсене $F$ 2-регулярно в точке $x_{0}$ относительно конуса $K$ по направлению $h$, причем $\|h\|<1$. Тогда для любого замкнутого выпуклого конуса $\widetilde{C}$ такого, что $\widetilde{C} \backslash\{0\} \subset \operatorname{ri} C$, июбого вектора $m \in \operatorname{ri} C$ найдется такое $b>0$, что отображение $F$ Г-накрывает в точке $x_{0}$ по кривой $\varphi$ относительно конуса $K$. Здесь семейство Г определяется по формуле (2), а кривая $\varphi-$ по формуле (10).

ДоКАЗАТЕЛЬСТвО этой теоремы получается дословньм повторением доказательства теоремы 2 , в которой лишь конус cone $B_{\delta}(l) \cap Y_{1}$ заменен на $\widetilde{C}$, а ссылка на теорему 1 - ссылкой на следствие 3 к ней.

4. Теорема об обратной функции и другие приложения. Всякая теорема о накрьвании по сути является теоремой об обратной функции, понимаемой как утверждение, гарантирующее существование решения нелинейного уравнения в окрестности заданного решения, причем с заданной оценкой. Реализуя это соображение, приведем соответствующую теорему об обратной функции, порожденную теоремой 2 . Итак, будем решать уравнение $F(x)=y$ относительно неизвестного $x \in\left(x_{0}+K\right)$ для $y$, принадлежащих окрестности точки $y_{0}$. 
Через $\rho(y, \widetilde{C})$ обозначим расстояние от точки $y$ до множества $\widetilde{C}$. Отметим, что если $\widetilde{C}$ - вьпуклый конус, то по теореме двойственности для задачи о кратчайшем расстоянии $[1$, с. 276$]$

$$
\rho(y ; \widetilde{C})=\sup \left\{\left\langle y^{*}, y\right\rangle, \quad y^{*} \in \widetilde{C}^{0}, \quad\left\|y^{*}\right\| \leqslant 1\right\},
$$

где $\widetilde{C}^{0}=\left\{y^{*} \in Y^{*}:\left\langle y^{*}, y\right\rangle \leqslant 0 \forall y \in \widetilde{C}\right\}$ - поляра конуса $\widetilde{C}$.

Предположим, что подпространство $Y_{1}=\operatorname{Lin} C$ замкнуто, топологически дополняемо и ri $C \neq \varnothing$.

Теорема 3 (об обратной функции). Предположим, что $h \in K$, отображение $F$ 2-регулярно в точке $x_{0}$ относительно конуса $K$ по направлению $h$ и выполняется (14). Тогда для любого $l \in \operatorname{ri} C$ найдутся такие положительные $\alpha, \beta$, что

$$
\begin{gathered}
\forall y \in Y:\left\|y-y_{0}\right\| \leqslant \alpha \quad \exists x=x(y) \in\left(x_{0}+K\right): F(x(y))=y, \\
\left\|x(y)-x_{0}\right\| \leqslant \operatorname{const}\left(\left\|y-y_{0}\right\|+\rho\left(y-y_{0} ; \widetilde{C}\right)^{1 / 2}\right), \quad \widetilde{C}=\operatorname{cone} B_{\beta}(l) \cap Y_{1} .
\end{gathered}
$$

Здесь и ниже использованная запись означает существование константы const, для которой справедливо выписанное неравенство.

ДокАЗАТЕЛьСтво. Используем обозначения и предположения, введенные при доказательстве теоремы 2. Соотношения (14) положительно однородны по $h$. Поэтому, не теряя общности, будем считать, что $\|h\|<1$. Возьмем $\delta>0$, отвечающеевекторам $h, l$ в силу теоремы 2 при $m=-Q[h, h]$. Положим $\widetilde{C}=\operatorname{cone} B_{\beta}(l) \cap Y_{1}, \beta=\frac{1}{3} \delta, \rho(y)=\rho(y ; \widetilde{C})$. По условию $l \in \operatorname{ri} \widetilde{C}$. Поэтому $\exists a_{1}>0:\left\langle y^{*}, l\right\rangle \leqslant-a_{1} \forall y^{*} \in Y_{1}^{*} \cap \widetilde{C}^{0}:\left\|y^{*}\right\|=1$. Покажем, что

$$
y_{1}+t l \in \widetilde{C} \quad \forall t \geqslant \tilde{\rho}\left(y_{1}\right)=a_{1}^{-1} \rho\left(y_{1}\right) \quad \forall y_{1} \in Y_{1} .
$$

Действительно, пусть $y \in Y_{1}$. Тогда $y \in \widetilde{C} \Longleftrightarrow\left\langle y^{*}, y\right\rangle \leqslant 0 \forall y^{*} \in Y_{1}^{*} \cap \widetilde{C}^{0}$. В то же время для $y^{*} \in Y_{1}^{*} \cap \widetilde{C}^{0},\left\|y^{*}\right\|=1$ и $t \geqslant \tilde{\rho}\left(y_{1}\right)$ имеем

$$
\left\langle y^{*}, y_{1}+t l\right\rangle \leqslant\left\langle y^{*}, y_{1}\right\rangle+\rho\left(y_{1}\right) a_{1}^{-1}\left\langle y^{*}, l\right\rangle \leqslant\left\langle y^{*}, y_{1}\right\rangle-\rho\left(y_{1}\right) \leqslant 0
$$

в силу (15), что и доказьвает (17).

Положим $D=$ cone $B_{\delta}(l) \cap Y_{1}$. По теореме 2 существуют такие $r_{1}>0, r_{2}>0, \varepsilon_{0} \leqslant 1$, что для любых $y_{2} \in Y, \tilde{y} \in D:\left\|y_{2}\right\| \leqslant r_{2},\|\tilde{y}\| \leqslant r_{2}, \varepsilon \in\left(0, \varepsilon_{0}\right)$ уравнение

$$
F(x)=y=\varepsilon \tilde{y}+\varepsilon^{2} y_{2}
$$

имеет решение $x \in\left(x_{0}+K\right):\left\|x-x_{0}\right\| \leqslant \varepsilon r_{1}$.

Обозначим через $\pi_{1}$ и $\pi_{2}$ непрерьвные операторы проектирования $Y$ на $Y_{1}$ и на некоторое замкнутое подпространство $Y_{2}$, дополняющее $Y_{1}$, соответственно. Отображения $\tilde{\rho}, \pi_{1}, \pi_{2}$ непрерьвны и $\tilde{\rho}(0)=0$. Поэтому $\exists \alpha>0$ :

$$
\left(\tilde{\rho}\left(\pi_{1} y\right) 2 r_{2}^{-1}\|l\|\right)^{1 / 2}+2 r_{2}^{-1}\left\|\pi_{1} y\right\|+\left(\left\|\pi_{2} y\right\| 2 r_{2}^{-1}\right)^{1 / 2} \leqslant \varepsilon_{0} \quad \forall y:\|y\| \leqslant \alpha .
$$

Взяв произвольньй вектор $y \in B_{\alpha}$, представим его в виде

$$
y=\varepsilon \tilde{y}+\varepsilon^{2} y_{2}, \quad y_{2}=-r_{2}(2\|l\|)^{-1} l+w
$$


где

$$
\begin{gathered}
\varepsilon=\varepsilon(y)=\max \left\{\left(\tilde{\rho}\left(\pi_{1} y\right) 2 r_{2}^{-1}\|l\|\right)^{1 / 2}, \quad 2 r_{2}^{-1}\left\|\pi_{1} y\right\|, \quad\left(\left\|\pi_{2} y\right\| 2 r_{2}^{-1}\right)^{1 / 2}\right\}, \\
w=\varepsilon^{-2} \pi_{2} y \in Y_{2}, \quad \tilde{y}=\varepsilon^{-1}\left(\pi_{1} y+\varepsilon^{2} r_{2}(2\|l\|)^{-1} l\right) .
\end{gathered}
$$

При этом, если $l=0$, то выражение $l\|l\|^{-1}$ условимся считать равным нулю. Докажем, что

$$
\varepsilon \leqslant \varepsilon_{0}, \quad \tilde{y} \in D, \quad\|\tilde{y}\| \leqslant r_{2}, \quad\left\|y_{2}\right\| \leqslant r_{2} .
$$

Действительно, первоенеравенство непосредственно вытекает из (19). Далее, если $l \neq 0$, то в силу $(21) \varepsilon^{2} r_{2}(2\|l\|)^{-1} \geqslant \tilde{\rho}\left(\pi_{1} y\right)$, откуда в силу (17) $\tilde{y} \in D$. Если же $l=0$, то $D=Y_{1} \Longrightarrow \tilde{y} \in D$. Далее, в силу $(21)\left\|\pi_{1} y\right\| \varepsilon^{-1} \leqslant r_{2} / 2 \Longrightarrow\|\tilde{y}\| \leqslant r_{2} / 2+\varepsilon r_{2} / 2 \leqslant r_{2}$. Наконец, в силу (21) $\left\|\pi_{2} y\right\| \varepsilon^{-2} \leqslant r_{2} / 2 \Longrightarrow\|w\| \leqslant r_{2} / 2$, что (см. (20)) дает последнее неравенство из (22).

Таким образом, в силу (20)-(22) уравнение (18) имеет решение $x \in\left(x_{0}+K\right):\left\|x-x_{0}\right\|$ $\leqslant \varepsilon r_{1}$. Последнее неравенство в силу $(21)$ и с учетом того, что $\tilde{\rho}\left(\pi_{1} y\right) \leqslant \tilde{\rho}(y)+$ const $\left\|\pi_{2} y\right\|$, $\left\|\pi_{2} y\right\| \leqslant \operatorname{const} \rho(y) \forall y$, доказывает оценку из (16). Теорема доказана.

ЗАмЕчаниЕ 1 . Пусть конус $C$ есть прямая сумма конечнопорожденного конуса $C_{1}$ и замкнутого подпространства $Y_{1}$, т.е. $C=C_{1}+Y_{1}$, причем $\left\|c_{1}\right\| \leqslant$ const $\left\|c_{1}+y_{1}\right\|$ $\forall c_{1} \in C_{1}, y_{1} \in Y_{1}$. Тогда теоремы 2 и 3 справедливы при $\widetilde{C}=C$.

ЗАмЕчАниЕ 2. Пусть для вектора $h \in K$ отображение $F$ 2-регулярно в точке $x_{0}$ относительно $K$ по направлению $h$, однако условие (14) нарушается. В этом случае уравнение $F(x)=y$ также имеет решения $x \in x_{0}+K$, но уже не в окрестности точки $y_{0}$, a $(m \in \operatorname{ri} C$ задано) в окрестности кривой (10). При этом также справедлива линейно-корневая оценка, аналогичная (16). Соответствующая точная формулировка приведена в [10].

В [11] для уравнения с конечномерным образом $Y$ получены условия, идентичные 2-регулярности, и их аналоги высших порядков, гарантирующие накрывание вдоль лучей. Одним из главных отличий результатов [11] от приведенных здесь является то, что в [11] доказана лишь локальная открытость соответствующего отображения, но без оценок типа (16).

ТеОрема 3'. Пусть пространство $Y$ конечномерно. Предполохим, что $h \in K$, отобрахсение $F$ 2-регулярно в точке $x_{0}$ относительно конуса $K$ по направлению $h$ и выполняется (14). Тогда для любого замкнутого выпуклого конуса $\widetilde{C}: \widetilde{C} \backslash\{0\} \subset \operatorname{ri} C$ найдется положительное $\alpha$, для которого выполняется (16).

Это утверждение вытекает из теорем $3,2^{\prime}$ и соображений компактности, применительно к единичной сфере конечномерного пространства.

Перейдем к теореме о неявной функции. Пусть $Z$ - банаховопространство и $F: X \times Z$ $\rightarrow Y$ - отображение, которое дважды непрерьвно дифференцируемо по Фреше в окрестности точки $\left(x_{0}, z_{0}\right)$, а его вторая производная липшицева. Положим $y_{0}=F\left(x_{0}, z_{0}\right)$. Рассмотрим уравнение $F(x, z)=y$ относительно неизвестного $x$ и в окрестности точки $\left(x_{0}, y_{0}, z_{0}\right)$ будем искать его решение $x=x(y, z)$. Положим

$$
C=\frac{\partial F}{\partial x}\left(x_{0}, z_{0}\right)(K)
$$

и пусть $Y_{1}=\operatorname{Lin} C$ замкнуто, топологически дополняемо и $\operatorname{ri} C \neq \varnothing$. 
Теорема 4 (о неявной функции). Предположим, ито $h \in K$, отображение $F$ 2-регулярно в точке $\left(x_{0}, z_{0}\right)$ относительно конуса $K$ по направлению $h$, т.е. отображсение $F\left(\cdot, z_{0}\right): X \rightarrow Y$ 2-регулярно в точке $x_{0}$ относительно конуса $K$ по направлению $h, u$

$$
\frac{\partial F}{\partial x}\left(x_{0}, z_{0}\right) h=0, \quad\left(-\frac{\partial^{2} F}{\partial x^{2}}\left(x_{0}, z_{0}\right)[h, h]\right) \in \operatorname{ri} C .
$$

Тогда для любых $l \in \operatorname{ri} C, q \in Z$ найдутся такие $\alpha, \beta>0$, что

$$
\begin{gathered}
\forall y, z:\left\|y-y_{0}\right\|+\left\|z-z_{0}\right\|<\alpha \quad \exists x=x(y, z) \in\left(x_{0}+K\right): F(x(y, z), z)=y \\
\left\|x(y, z)-x_{0}\right\| \leqslant \operatorname{const}\left(\left\|y-y_{0}\right\|+\left\|z-z_{0}\right\|+\left(\left\|\pi_{2}\left(y-y_{0}-\frac{\partial F}{\partial z}\left(x_{0}, z_{0}\right)\left(z-z_{0}\right)\right)\right\|\right.\right. \\
\left.\left.+\rho\left(\left(\pi_{1}\left(y-y_{0}\right), z-z_{0}\right), \operatorname{cone} B_{\beta}(v)\right)\right)^{1 / 2}\right) .
\end{gathered}
$$

Здесь $v=\left(l+\pi_{1}(\partial F / \partial z)\left(x_{0}, z_{0}\right) q, q\right) \in Y \times Z, a \pi_{1} u \pi_{2}($ как и выше $)$ - непрерывные операторы проектирования $Y$ на $Y_{1}$ и на замкнутое подпространство $Y_{2}$, дополняющее $Y_{1}$, соответственно.

ДоКАЗАТЕЛЬСтво. Пусть для простоты $y_{0}=0, z_{0}=0$. Превратим $Y \times Z$ в банахово, определив в нем норму как сумму норм в $Y$ и $Z$. Будем решать систему уравнений $F(x, z)=y, z=\mu$ относительно неизвестных $(x, z)$ в окрестности точки $\left(\left(x_{0}, z_{0}\right)\right.$, $\left.\left(y_{0}, \mu_{0}\right)\right)$, где $\mu_{0}=z_{0}$. Для этого рассмотрим отображение $\mathscr{F}: X \times Z \rightarrow Y \times Z$, $\mathscr{F}(x, z)=(F(x, z), z)$. Очевидно, $\left(h, 0_{z}\right) \in \operatorname{Ker} \mathscr{F}^{\prime}\left(x_{0}, z_{0}\right) ;$

$$
-\mathscr{F}^{\prime \prime}\left(x_{0}, z_{0}\right)\left[\left(h, 0_{z}\right),\left(h, 0_{z}\right)\right]=\left(m, 0_{z}\right) \in \operatorname{ri} W .
$$

Здесь

$$
m=-\frac{\partial^{2} F}{\partial x^{2}}\left(x_{0}, z_{0}\right)[h, h], \quad W=\mathscr{F}^{\prime}\left(x_{0}, z_{0}\right)(K \times Z),
$$

а $0_{z}$ - нулевой элемент пространства $Z$. Ясно, что

$$
W=\left\{(y, z): y=\tilde{y}+\frac{\partial F}{\partial z}\left(x_{0}, z_{0}\right) z, \quad \tilde{y} \in C, \quad z \in Z\right\}
$$

и, значит, Lin $W$ замкнуто. Поэтому к отображению $\mathscr{F}$ применима теорема 3 об обратной функции, дающая искомое утверждение. При этом использовано следующее соображение.

Положим $A=(\partial F / \partial x)\left(x_{0}, z_{0}\right), w=(l+A q, q)$ и $D=\operatorname{cone} B_{\beta}(w) \cap \operatorname{Lin} W$, где $\beta>0$ взято из теоремы 3 . Возьмем произвольные $y, z$. Имеем

$$
\rho((y, z), D) \leqslant\left\|(y, z)-\left(y_{1}, z\right)\right\|+\rho\left(\left(y_{1}, z\right), D\right), \quad \text { где } y_{1}=\pi_{1} y+\pi_{2} A z .
$$


Очевидно, $\left(y_{1}, z\right) \in \operatorname{Lin} W$, так как $\pi_{2} A z=A z-\pi_{1} A z$. Поэтому

$$
\begin{aligned}
\rho\left(\left(y_{1}, z\right), D\right) & =\rho\left(\left(y_{1}, z\right), \operatorname{cone} B_{\beta}(w)\right)=\inf _{t \geqslant 0} \max \left(0,\left\|\left(y_{1}, z\right)-t w\right\|-t \beta\right) \\
& =\inf _{t \geqslant 0} \max \left(0,\left(\left\|y_{1}-t(l+A q)\right\|+\|z-t q\|-t \beta\right)\right) \\
& =\inf _{t \geqslant 0} \max \left(0,\left\|\pi_{2} A(z-t q)+\pi_{1} y-t\left(\pi_{1} A q+l\right)\right\|+\|z-t q\|-t \beta\right) \\
& \leqslant \inf _{t \geqslant 0} \max \left(0,\left(\left\|\pi_{2} A\right\|+1\right)\|z-t q\|+\left\|\pi_{1} y-t\left(\pi_{1} A q+l\right)\right\|-t \beta\right) \\
& \leqslant \inf _{t \geqslant 0} \max \left(0, c\|z-t q\|+c\left\|\pi_{1} y-t\left(\pi_{1} A q+l\right)\right\|-t \beta\right) \\
& =c \rho\left(\left(\pi_{1} y, z\right), \operatorname{cone} B_{\tilde{\beta}}(v)\right), \quad \text { где } c=1+\left\|\pi_{2} A\right\| \geqslant 1, \quad \tilde{\beta}=\frac{\beta}{c} .
\end{aligned}
$$

Учитьвая, что $y-y_{1}=\pi_{2}(y-A z)$, окончательно получаем

$$
\rho((y, z), D) \leqslant\left\|\pi_{2}(y-A z)\right\|+c \rho\left(\left(\pi_{1} y, z\right), \operatorname{cone} B_{\tilde{\beta}}(v)\right) .
$$

Теорема доказана.

ЗАмЕчАнИЕ. Пусть пространства $Y, Z$ конечномерны и $\widetilde{C}$ - вьпукльй замкнутьй конус, для которого $\widetilde{C} \backslash\{0\} \subset$ ri $C$. Предположим, что вьполнены предположения теоремы 4. Тогда найдутся такие положительные $\alpha$ и $\beta$, что для любых $l \in \operatorname{ri} \widetilde{C}, q \in Z$ выполняется (23). Справедливость этого утверждения вытекает из теоремы 4 и соображений компактности конечномерной сферы.

Зададимся вопросом: что будет, если в предположениях теоремы о неявной функции условие

$$
\frac{\partial^{2} F}{\partial x^{2}}\left(x_{0}, z_{0}\right)[h, h] \in-\operatorname{ri} C
$$

нарушается. Оказьвается, что и в этом случае уравнение $F(x, z)=y$ можно разрешить, но уже не для всех $(y, z)$, близких к исходной точке $\left(y_{0}, z_{0}\right)$. Приведем соответствующее утверждение.

ТЕОрема 5 (о неявной функции). Предположим, ито $h \in K$, отображсене $F$ 2-регулярно в точке $\left(x_{0}, z_{0}\right)$ относительно конуса $K$ по направлению $h u$

$$
\frac{\partial F}{\partial x}\left(x_{0}, z_{0}\right) h=0, \quad \frac{\partial^{2} F}{\partial x^{2}}\left(x_{0}, z_{0}\right)[h, h] \in \operatorname{Lin} C .
$$

Тогда для любых $l \in \operatorname{ri} C, q \in Z, \gamma>0$ найдутся такие $\alpha, \beta>0$, что

$$
\begin{aligned}
& \forall(y, z) \in\left(y_{0}, z_{0}\right)+\operatorname{cone} B_{\beta}\left(\left(l+\frac{\partial F}{\partial z}\left(x_{0}, z_{0}\right) q, q\right)\right) \cap B_{\alpha}: \\
& \left\|\pi_{2}\left(y-y_{0}-\frac{\partial F}{\partial z}\left(x_{0}, z_{0}\right)\left(z-z_{0}\right)\right)\right\| \\
& \leqslant \gamma\left(\left\|\pi_{1}\left(y-y_{0}\right)+\pi_{2} \frac{\partial F}{\partial z}\left(x_{0}, z_{0}\right)\left(z-z_{0}\right)\right\|^{2}+\left\|z-z_{0}\right\|^{2}\right) \\
& \exists x=x(y, z) \in\left(x_{0}+K\right): F(x(y, z), z)=y, \\
& \left\|x(y, z)-x_{0}\right\| \leqslant \operatorname{const}\left(\left\|y-y_{0}\right\|+\left\|z-z_{0}\right\|\right) .
\end{aligned}
$$


Доказательство аналогично доказательству теорем $2,4$.

При $K=X$ теоремы о неявной функции в анормальной точке при аналогичных предположениях доказаны в [4], [8], а также в предположениях иного характера в [12], [9], причем в последней работе в оценке на решение (23) вследствие опечатки на с. 7 в последнем члене, стоящем под корнем и имеющем множитель $P_{2}$, пропущено слагаемое $(\partial F / \partial y)\left(x_{0}, y_{0}\right)\left(y-y_{0}\right)$. При выполнении условия Робинсона $(\partial F / \partial x)\left(x_{0}, z_{0}\right)(K)=Y$ теоремы 3,4 получены в [2]. Отметим, что в теоремах $3,3^{\prime}$ и 4 замкнутость конуса $C$ априори не предполагается и это существенно, так как в приложениях часто $C$ не замкнут. Так, например, в классической задаче оптимального управления вычисления показывают, что в общем положении конус $C \backslash\{0\}$ открыт (при этом $Y$ конечномерно)!

При исследовании структуры множества уровня вида $M=\left\{x \in x_{0}+K: F(x)=\right.$ $\left.F\left(x_{0}\right)\right\}$ в окрестности точки $x_{0}$ большое значение имеет удобное описание конуса касательных к нему в точке $x_{0}$ векторов. Напомним, что для множества $M \subset X$ вектор

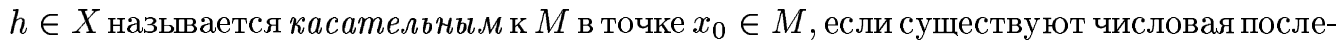
довательность $\left\{\varepsilon_{i}\right\} \rightarrow 0+$ и сходящаяся к нулю последовательность векторов $\left\{\tilde{x}_{i}\right\}$, для которых $x_{0}+\varepsilon_{i} h+\varepsilon_{i} \tilde{x}_{i} \in M \forall i$. Конус касательных к $M$ в точке $x_{0}$ векторов обозначается через $T M\left(x_{0}\right)$. Очевидно, $T M\left(x_{0}\right) \subseteq \operatorname{Ker} F^{\prime}\left(x_{0}\right) \cap K$. Более того, справедливо включение $T M\left(x_{0}\right) \subseteq H\left(x_{0}\right)$, где

$$
H\left(x_{0}\right)=\left\{h \in \operatorname{Ker} F^{\prime}\left(x_{0}\right): h \in K, \quad\left(-F^{\prime \prime}\left(x_{0}\right)[h, h]\right) \in \operatorname{cl} F^{\prime}\left(x_{0}\right)(K)\right\},
$$

a $\mathrm{cl}$ - замькание множества. Действительно, пусть $C=F^{\prime}\left(x_{0}\right)(K)$. Для $h \in T M\left(x_{0}\right)$ имеем

$$
\begin{aligned}
x_{i}=\varepsilon_{i} h+\varepsilon_{i} \tilde{x}_{i} \in K \quad \forall i & \Longrightarrow F^{\prime}\left(x_{0}\right) x_{i}+\frac{1}{2} F^{\prime \prime}\left(x_{0}\right)\left[x_{i}, x_{i}\right]=o\left(\varepsilon_{i}^{2}\right) \\
& \Longrightarrow\left\langle y^{*}, F^{\prime \prime}\left(x_{0}\right)[h, h]\right\rangle \geqslant 0 \quad \forall y^{*} \in C^{0} \\
& \Longrightarrow\left(-F^{\prime \prime}\left(x_{0}\right)[h, h]\right) \in C^{00}=\operatorname{cl} C
\end{aligned}
$$

Вообще говоря, $T M\left(x_{0}\right) \neq H\left(x_{0}\right)$, однако, если вьполняется условие Робинсона $F^{\prime}\left(x_{0}\right)(K)=Y$, то $T M\left(x_{0}\right)=H\left(x_{0}\right)$. Приведем достаточные условия касания, более тонкие, чем условие Робинсона. Их естественно назьвать достаточными условиями касания второго порядка в отличие от условия Робинсона, которое является условием первого порядка.

Теорема 6. Предположим, что $h \in K$, отображение $F$ 2-регулярно в точке $x_{0}$ относительно конуса $K$ по направлению $h$ и имеет место (14). Тогда $h \in T M\left(x_{0}\right)$.

ДокАЗАТЕЛЬСТво. Будем использовать обозначения, введенные при доказательстве теоремы 2 . Для $\varepsilon>0$ рассмотрим уравнение

$$
F\left(x_{0}+\varepsilon h+\varepsilon^{2} \eta+\varepsilon^{2} x_{1}+\varepsilon x_{2}\right)=0
$$

относительно неизвестных $x_{1} \in K, x_{2} \in K_{2}$, где $\eta \in K: F^{\prime}\left(x_{0}\right) \eta=-\frac{1}{2} F^{\prime \prime}\left(x_{0}\right)[h, h]$. Дословно повторяя рассуждения, проведенные при доказательстве теоремы 2 при $\tilde{y}=$ $y=0$, получаем, что рассматриваемое уравнение эквивалентно следующему (см. (13)):

$$
A\left(x_{1}, x_{2}\right)=\tilde{f}\left(x_{1}, x_{2} ; \varepsilon\right) .
$$


Здесь $\tilde{f}\left(x_{1}, x_{2} ; \varepsilon\right)=f\left(x_{1}, x_{2} ; 0,0, \varepsilon\right)$. По построению $\tilde{f}(0,0 ; \varepsilon) \rightarrow 0, \varepsilon \rightarrow 0$. Кроме того, $\gamma(r, \varepsilon) \rightarrow 0,(r, \varepsilon) \rightarrow 0$, где $\gamma(r, \varepsilon)$ - константа Липшица функции $\tilde{f}(\cdot ; \varepsilon)$ по переменным $x_{1}, x_{2}$ на шаре радиуса $r$. Отсюда в силу предложения 1 для произвольного натурального $i$ найдется такое $\varepsilon_{i}>0$, что рассматриваемое уравнение при $\varepsilon=\varepsilon_{i}$ имеет решение $x_{1, i} \in K, x_{2, i} \in K \cap \operatorname{Ker} F^{\prime}\left(x_{0}\right):\left\|x_{1, i}\right\|+\left\|x_{2, i}\right\| \leqslant r=i^{-1}$. Очевидно, $x_{1, i}, x_{2, i} \rightarrow 0$ и, значит, $h \in T M\left(x_{0}\right)$. Теорема доказана.

Приведем удобное для приложений (например, для теории экстремальных задач) обобщение теоремы 6 . Пусть $N$-замкнутьй выпукльй конус из $Y$. Рассмотрим множество

$$
M=\left\{x \in x_{0}+K: F(x) \in F\left(x_{0}\right)+N\right\} .
$$

Положим $C=F^{\prime}\left(x_{0}\right)(K)-N$. Пусть подпространство $Y_{1}=\operatorname{Lin} C$ замкнуто, топологически дополняемо и $\mathrm{ri} C \neq \varnothing$.

ТЕОрема 6'. Предполохим, что $h \in K$ и отобрахение $F$ 2-регулярно в точке $x_{0}$ относительно конусов $K$ и $N$ по направлению $h$, т.е. имеет место

$$
\begin{gathered}
F^{\prime}\left(x_{0}\right) h \in N, \quad\left(-F^{\prime \prime}\left(x_{0}\right)[h, h]\right) \in \operatorname{ri} C, \\
\operatorname{Lin} C+F^{\prime \prime}\left(x_{0}\right)\left[h, F^{\prime}\left(x_{0}\right)^{-1}(N) \cap K\right]=Y .
\end{gathered}
$$

Тогда $h \in T M\left(x_{0}\right)$, где мнохество $M$ определено выше.

ДокАЗАтЕльство. Определим отображение $\mathscr{F}: X \times Y \rightarrow Y$ по формуле $\mathscr{F}(x, y)=$ $F(x)-y$, конус $\mathscr{K}=\{(x, y): x \in K, y \in N\}$ и вектор $\tilde{h}=\left(h, F^{\prime}\left(x_{0}\right) h\right) \in \mathscr{K}$. Применяя к $\tilde{h}, \mathscr{K}$ и отображению $\mathscr{F}$ в точке $\left(x_{0}, y_{0}\right)$ теорему 6 , получаем требуемое. Теорема доказана.

ЛЕмма 2. Предположими, что $h \in K, F^{\prime}\left(x_{0}\right) h \in N,\left(-F^{\prime \prime}\left(x_{0}\right)[h, h]\right) \in C$ и имеет место (25). Пусть, кроме того,

$$
\exists \tilde{h} \in K: F^{\prime}\left(x_{0}\right) \tilde{h} \in N, \quad\left(-F^{\prime \prime}\left(x_{0}\right)[h, \tilde{h}]\right) \in \operatorname{ri} C .
$$

Тогда существует такая, сходящаяся $к$ нулю последовательность $\left\{x_{i}\right\}$, что для $h_{i}=h+i^{-1} \tilde{h}+i^{-1} x_{i}$ выполняется

$$
h_{i} \in K, \quad F^{\prime}\left(x_{0}\right) h_{i} \in N, \quad F^{\prime \prime}\left(x_{0}\right)\left[h_{i}, h_{i}\right] \in \operatorname{Lin} C
$$

$u$, следовательно, при больиих $i$ отображсние $F$ 2-регулярно в точке $x_{0}$ относительно конуса $K$ по направлению $h_{i}$.

ДоказАтельСтво. Рассмотрим множество $\widetilde{M}=\left\{x \in \widetilde{K}: \pi_{2} F^{\prime \prime}\left(x_{0}\right)[x, x]=0\right\}$, где $\widetilde{K}=\left\{x \in K: F^{\prime}\left(x_{0}\right) x \in N\right\}$. Очевидно, $h \in \widetilde{M}$, а $\tilde{h}$ является касательным к $\widetilde{M}$ в этой точке $h$ (например, по теореме 6). Поэтому существует сходящаяся к нулю последовательность $\left\{x_{i}\right\}$, для которой выполняется $h_{i}=h+i^{-1} \tilde{h}+i^{-1} x_{i} \in \widetilde{M}$ и, значит, имеет место (26). Лемма доказана.

Если в (24) последнее условие ослабить, заменив ri $C$ на $C$, то вектор $h$ касательным к $M$, вообще говоря, не будет. Тем не менее, если для $h$ вьполнены предположения 
леммы 2 , то $h \in T M\left(x_{0}\right)$. Действительно, для больших номеров $i$ векторы $h_{i}$ удовлетворяют предположениям теоремы $6^{\prime}$ и, следовательно, $h_{i} \in T M\left(x_{0}\right)$, а касательный конус замкнут. Теоремы 6 и $6^{\prime}$ обобщают некоторые результаты из [4], [12], [13], а в предположении выполнении условия Робинсона $F^{\prime}\left(x_{0}\right)(K)-N=Y$ они установлены в [2].

Я благодарю профессоров Е. Р. Авакова, А.Ф. Измаилова, А. Д. Иоффе, М.Ф. Сухинина и В. М. Тихомирова за ценные советы и полезные обсуждения.

\section{СПИСОК ЦИТИРОВАННОЙ ЛИТЕРАТУРЫ}

[1] Алексеев В. М., Тихомиров В. М., Фомин С. В. Оптимальное управление. М.: Наука, 1979.

[2] Robinson S. Stability theory for Systems of inequalities. Part II: differentiable nonlinear systems // SIAM J. Numer. Anal. 1976. V. 13. №4. P. 497-513.

[3] Дмитрук А. В., Милютин А. А., Осмоловский Н. П. Теорема Люстерника и теория экстремума // УМН. 1980. Т. 35. №6. С. 11-46.

[4] Аваков Е.Р. Теоремы об оценках в окрестности особой точки отображения // Матем. заметки. 1990. Т. 47. №5. С. 3-13.

[5] Обен ЖК. П., Экланд И. Прикладной нелинейный анализ. М.: Мир, 1988.

[6] Колмогоров А. Н., Фомин С. В. Элементы теории функций и функционального анализа. М.: Наука, 1989.

[7] Экланд И., Темам Р. Выпуклый анализ и вариационные проблемы. М.: Мир, 1979.

[8] Измаилов А. $\Phi$. Теоремы о представлении семейств нелинейных отображений и теоремы о неявной функции // Матем. заметки. 2000. Т. 67. № 1. С. 57-68.

[9] Арутюнов А. В. Теорема о неявной функции как реализация принципа Лагранжа. Анормальные точки // Матем. сб. 2000. Т. 191. № 1. С. 3-26.

[10] Арутюнов А. В. Теорема об обратной функции на конусе в окрестности анормальной точки // Докл. РАН. 2003. Т. 389. №1. С. 7-10.

[11] Warga J. Higher-order conditions for conical controllability // SIAM J. Control Optimiz. 1988. V. 26. №6. P. 1471-1480.

[12] Измаилов А.Ф., Третьяков А. А. 2-регулярные решения нелинейных задач. М.: Физматлит, 1999.

[13] Измаилов А.Ф. К условиям оптимальности в экстремальных задачах с нерегулярными ограничениями-неравенствами // Матем. заметки. 1999. Т. 66. №1. С. 89-102.

Российский университет Дружбы народов, г. Москва

Поступило

01.03.2004 